\title{
Quantum Interference Noise Near the Dirac Point in Graphene
}

\author{
Atikur Rahman, Janice Wynn Guikema, and Nina Marković \\ Department of Physics and Astronomy, Johns Hopkins University, Baltimore, Maryland 21218, USA.
}

\begin{abstract}
Effects of disorder on the electronic transport properties of graphene are strongly affected by the Dirac nature of the charge carriers in graphene. This is particularly pronounced near the Dirac point, where relativistic charge carriers cannot efficiently screen the impurity potential. We have studied time-dependent conductance fluctuations and magnetoresistance in graphene in the close vicinity of the Dirac point. We show that the fluctuations are due to the quantum interference effects due to scattering on impurities, and find an unusually large reduction of the relative noise power in magnetic field, possibly indicating that an additional symmetry plays an important role in this regime.
\end{abstract}

In disordered electronic systems, quantum corrections to the conductance arise due to quantum interference between paths of electrons scattered on random impurities. In the absence of a magnetic field, the electron paths that traverse the loops in a clockwise fashion interfere constructively with the counterclockwise paths through the same loops, resulting in a small change in the conductance. Specifically, the backscattered paths (the paths that return to their origin) lead to a correction to the average conductance of the system, known as weak localization (WL) 1 3. Magnetic field adds a different phase factor to the paths that are identical, but traversed in the opposite sense, removing the WL corrections, but one still observes the universal conductance fluctuations (UCF) as a function of magnetic field or chemical potential, which arise from adding the interference contributions from all possible paths [4, 5]. The quantum interference contribution to the conductance also fluctuates if the impurity configuration changes over time, leading to time-dependent conductance fluctuations that are expected to cause $1 / f$ noise [6 6 ]

In graphene, the quantum interference phenomena are affected by the pseudospin and valley degrees of freedom 9, 10. Conservation of pseudospin precludes backscattering, suppressing WL and causing weak antilocalization (WAL) 11], while intervalley scattering restores the WL [12 14]. Additional effects, such as defects and corrugations, can completely suppress the quantum corrections [15]. Depending on the carrier density and the nature of the disorder, all three regimes (WL, WAL and the suppression of quantum corrections) are observed experimentally [16, 17]. UCF in graphene depend on the carrier density and the nature of the impurity scattering, but can also depend on the details and the geometry of the sample [18 20. In particular, strong intervalley scattering is found to suppress UCF [18, 19, in contrast to its effect on WL. However, the majority of the theoretical and experimental work on quantum corrections has focused on the regime away from the Dirac point. In the close vicinity to the Dirac point (at low doping and low temperatures), the relativistic Dirac quasiparticles are unable to screen the long-range Coulomb interactions in the usual way,
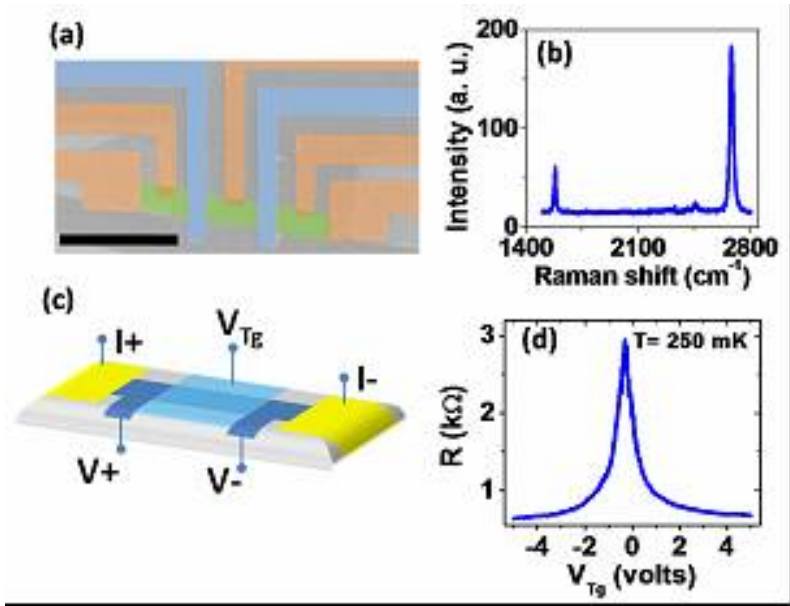

FIG. 1: (a) False color scanning electron microscope image of a typical single layer device (SL1). The graphene flake is highlighted in green and the top gates are shown in blue. The distance between the voltage leads is typically around one micrometer. The scale bar is $3 \mu \mathrm{m}$ long. (b) Raman spectra of one of the samples. The observed peak structure is characteristic for single layer graphene. (c) Schematic of the four probe measurement setup with external voltage probes. (d) Resistance as a function of top gate voltage for zero back gate voltage for sample SL1. The Dirac point, or the charge neutrality point, is located at $V_{T g}=-0.3 \mathrm{~V}$.

altering electron-electron interactions [21].

In this work, we describe measurements of the timedependent conductance fluctuations in graphene, focusing specifically on the low-carrier density regime near the Dirac point. We find that the $1 / f$ noise is reduced in magnetic field, with a characteristic field and temperature dependence that suggests quantum interference as the origin of the noise. However, the observed relative noise reduction is twice as large as what one might expect based on the fundamental symmetry considerations and the current theoretical understanding of quantum transport in graphene.

A scanning electron microscope image of a typical top gated device (SL1) is shown in Fig. 1(a) (see Supporting Information for fabrication details). Raman spectroscopy was used to confirm that all samples were single 


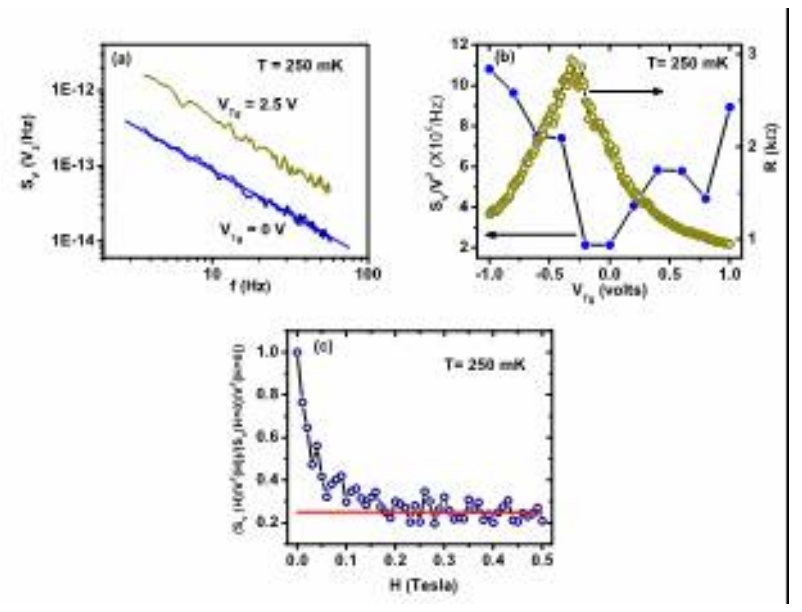

FIG. 2: (a) Noise power as a function of frequency (plotted on a log-log scale) for two different values of the top gate voltage. Straight line shows the $1 / \mathrm{f}$ dependence of the noise spectra. (b) Noise power (left) and resistance (right) as a function of top gate voltage in the vicinity of the Dirac point. (c) Normalized noise power as a function of magnetic field for a single layer graphene device. It is evident that the relative noise is reduced by a factor of four from its zero magnetic field value above a certain field. The straight line indicates the reduction of the zero-field value by a factor of four.

layer graphene, with typical results shown in Fig. 1(b). Electrical measurements were done in a 4-probe geometry, using external voltage probes [22] as shown on the schematic in Fig. 1(c). The typical resistance as a function of top gate voltage $\left(V_{T g}\right)$ with zero back gate voltage $\left(V_{B g}\right)$ is shown in Fig. 1(d). The peak in the resistance occurs at the Dirac point, at the value of top gate voltage $-0.3 \mathrm{~V}$, at which the carrier density in graphene reaches the minimum.

Low-frequency noise measurements were done using the ac noise measurement technique [23]. The measured noise power $\left(S_{V}\right)$ showed $1 / f^{\alpha}$ dependence for either gate (top or back) voltage with values of $\alpha$ close to 1 (Fig. $2(\mathrm{a})$ ). We found that the noise data were highly reproducible over time at any temperature and did not depend on the direction or scan step of the gate voltage. The normalized noise power density $\left(=f S_{V} / V^{2}\right.$ or $\left.f S_{I} / I^{2}\right)$ was found to be independent of the bias current or voltage (see Supporting information, Fig. S1), ruling out any issues due to heating by the bias current.

The normalized noise power as a function of the top gate voltage in the vicinity of the Dirac point is shown in Fig. 2(b). We find that the noise decreases upon approaching the Dirac point from both directions, reaching a minimum close to the Dirac point.

When a magnetic field is applied perpendicular to the substrate, the noise power decreases rapidly as shown in Fig. 2(c). After reaching some characteristic value of the field, the relative noise power saturates at the value that is a factor of four smaller than the zero-field

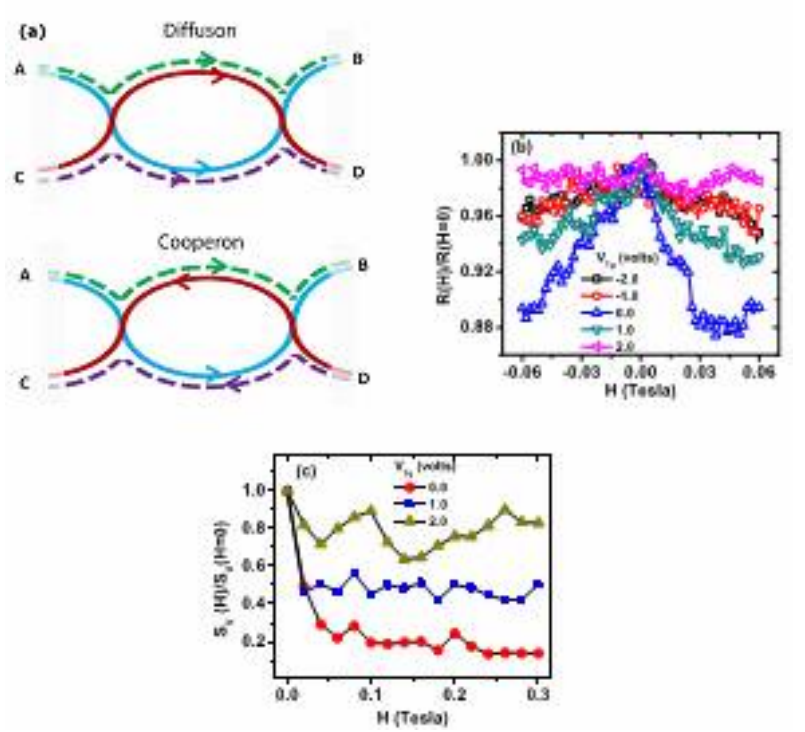

FIG. 3: (a) Schematic of pairs of electron trajectories that form closed loops. The conductance fluctuations are caused by interference between paths from $\mathrm{A}$ to $\mathrm{B}$ and those from $\mathrm{C}$ to $\mathrm{D}$ that intersect somewhere in the interior of the sample. The Diffuson contribution is shown in the upper panel: all the paths in the loop are traversed in the same sense, and the magnetic field does not introduce a relative phase factor. In the Cooperon channel, shown in the bottom panel, the magnetic field introduces a relative phase when the loop is traversed in the opposite sense. In this case, contributions from various loops no longer add in a coherent way and the quantum corrections to conductivity disappear. (b) Magnetoresistance as a function of magnetic field for various top gate voltages with zero back gate voltage (sample SL1). A negative magnetoresistance is observed in the vicinity of the Dirac point, with the maximum of magnetoresistance observed close to zero top gate. As the gate voltage is increased in both directions, the magnetoresistance decreases. (c) Relative noise power for different top gate voltages as a function of a magnetic field (sample SL1). The reduction of the relative noise power by about a factor of four is observed at zero top gate, but this factor decreases for larger values of top gate voltage.

value. Assuming that the characteristic field corresponds to threading one flux quantum through a phase-coherent area of the sample, we find the phase coherence length $\left(\mathrm{E}_{\phi}\right)$ to be in the range between $200-300 \mathrm{~nm}$ for our samples.

The low-frequency noise was studied experimentally in single-layer graphene transistors and was suggested to be related to the fluctuating charges in the vicinity of graphene [24 26]. However, the reduction of the noise power upon application of a small magnetic field observed here is a strong indication that quantum interference effects dominate the low-frequency noise. In the case of disordered metals, the conductance fluctuations can be calculated by considering all possible trajectories that an electron can take while scattering off of random impurities. Particularly important are the combinations of 
paths that connect two different points in a sample, as shown in Fig. 3(a). There are two contributions to the interference between paths from A to B and those from C to D: diffuson and cooperon contribution. The diffuson contribution is insensitive to the magnetic field, as no relative phase is introduced between the various paths. On the contrary, the magnetic field removes the cooperon contribution, reducing the number of conduction channels by a factor of two and reducing the relative noise by precisely a factor of two [8].

In the same regime, we find negative magnetoresistance as a function of magnetic field at different gate voltages, is shown in Fig. 3(b). Negative magnetoresistance may be due to WL, but we observe it only in the narrow range of gate voltages in the vicinity of the Dirac point - the negative magnetoresistance decreases as the gate voltage is increased in both directions. The effect of magnetic field on noise also depends on the gate voltage as shown in Fig. 3(c). Away from the Dirac point, the noise becomes less sensitive to the magnetic field for both positive and negative gate voltages. The noise characteristics are found to be symmetric with respect to magnetic field, and similar results were found for both back gate and top gate. The four-fold reduction in the noise is not always observed precisely at the Dirac point, but it coincides exactly with the gate voltage at which the maximum negative magnetoresistance is also observed (at small positive gate voltages relative to the Dirac point). Similar behavior is observed as a function of back gate voltage (see Supporting information, Fig. S2). The overall change in resistance is small compared to the change in the noise (see Supporting information, Fig. S3).

According to the present understanding, WL can be observed in graphene in the presence of strong intervalley scattering, which can arise due to atomically sharp potentials (such as edges or atomic defects). In the case of strong intervalley scattering, many aspects of quantum transport in graphene are expected to be identical to those in disordered metals [10, 14, 18, 19]. In particular, the conductance fluctuations should exhibit universal properties, as they depend only on the symmetries of the random ensembles that describe the disordered system, and not on their detailed configuration. The variance of the interference-induced conductance fluctuations in graphene will generally have a prefactor that depends on the interplay of inelastic and elastic scattering lengths and the shape of the sample [18, 19, but graphene with broken valley symmetry should belong to the orthogonal Wigner-Dyson symmetry class in the absence of a magnetic field [10]. Application of a magnetic field will put it in the unitary symmetry class, and a two-fold reduction in the relative noise power will be expected on general symmetry grounds.

Additional two-fold reduction in the relative noise power is expected when the Zeeman energy exceeds
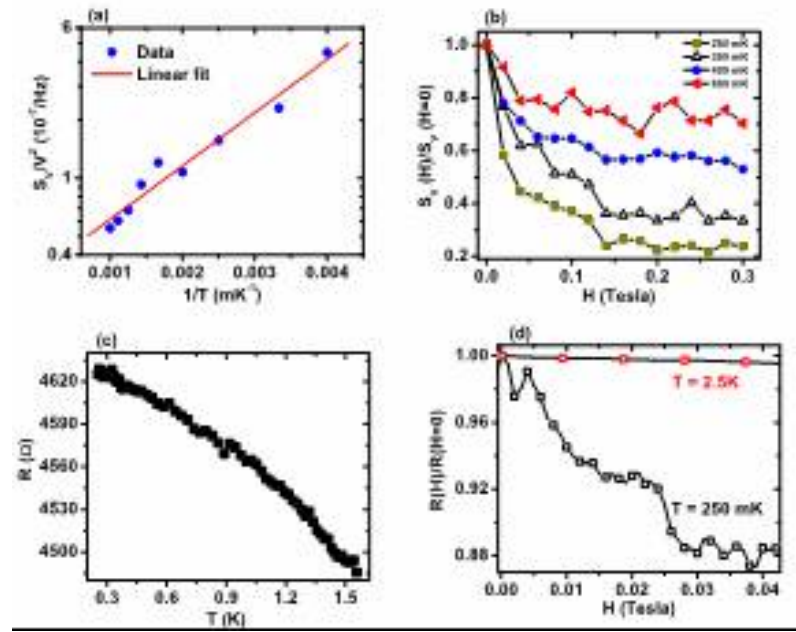

FIG. 4: (a) Normalized noise power is shown as a function of inverse temperature on a log-linear scale. The line represents a linear fit, and the normalized noise power shows a $\exp (1 / T)$ dependence on temperature. (b) Relative noise power is plotted as a function of magnetic field for different temperatures. The reduction of the relative noise power by a factor of four is observed at $250 \mathrm{mK}$, but the reduction is smaller at higher temperatures. (c) Resistance as a function of temperature is shown for $V_{T g}, V_{B g}=0$, in the regime highlighted in Fig. 2 (b). The slight increase of the resistance with decreasing temperature indicates insulating behavior. (d) Magnetoresistance as a function of magnetic field is shown for two different temperatures. It is evident that a much smaller magnetoresistance is observed at higher temperatures.

$h D / L_{\phi}^{2}$ [8]. The two-fold reduction in the noise power has been observed in metals [27, 28, as well as the additional two-fold reduction at a larger magnetic field due to Zeeman splitting [29, 30. In our samples, the Zeeman splitting cannot explain the four-fold reduction, which is observed for small characteristic fields $(50 \mathrm{mT})$, where the Zeeman splitting $(0.006 \mathrm{meV})$ is smaller than both the thermal energy $(0.02 \mathrm{meV})$ and $h D / L_{\phi}^{2}(0.08 \mathrm{meV})$.

The temperature dependence of the noise is also unusual in this regime. For normal metals in the phase coherent regime, the noise due to fluctuating scatterers depends on temperature as $T^{-1}$, as observed in several systems [27, 28. We found that the noise decreases with increasing temperature and the normalized noise power shows a $\exp (1 / T)$ dependence, as shown in Fig. 4(a) (similar dependence was also found in other work [31]). As the temperature increases, the relative noise power is still reduced in magnetic field, but by a smaller factor, as shown in Fig. 4(b). The sample resistance increases slightly with decreasing temperature, as shown in Fig. 4(c), so the temperature dependence of the noise cannot be explained by the resistance change. The slowly increasing resistance with decreasing temperature is consistent with $\mathrm{WL}$, as is the fact that the negative magnetoresistance also decreases with increasing temperature, as shown in 
Fig. 4(d).

It is well known that charge-inhomogeneous regions (puddles) tend to form in the vicinity of the Dirac point 32. The presence of the top gate also locally dopes the graphene, forming pn junctions at the edges. A random network of puddles or pn junctions could be expected to show conductance fluctuations and magnetoresistance that reflect the fluctuations in the electrostatic environment [33, 34]. However, one might expect such fluctuations to increase with temperature, leading to the increase of the noise power with increasing temperature, which is in contradiction to our observations. The temperature dependence of the resistance, magnetoresistance and the relative noise power reduction in magnetic field are all consistent with a decrease of the phase coherence length as the temperature is increased. In addition, the observation of the Aharonov-Bohm oscillations in similar samples confirms that both the electron and the hole transport is phase-coherent across pn junctions and any electron-hole puddles in the vicinity of the Dirac point 35 .

The decrease of the quantum interference noise by a factor of four in magnetic field is not presently understood, but the unusual nature of quantum corrections near the Dirac point may offer insight into phenomena observed in other experiments, such as the fourfold decrease of mobility depending on the nature of impurity scattering 36, or the anomalous backscattering [37, 38] near the Dirac point. A better understanding of the quantum interference noise may also provide useful clues about the nature of the impurity scattering in this regime.

The authors would like to thank A. Morpurgo, E. McCann, F. Guinea, V. Fal'ko and I. Aleiner for useful comments and suggestions. N. M. would like to thank the Aspen Center for Physics and the NSF Grant 1066293. J. W. G. was supported in part by the M. Hildred Blewett Fellowship of the American Physical Society.

[1] B. L. Altshuler, D. Khmelńitzkii, A. I. Larkin, and P. A. Lee, Phys. Rev. B 22, 5142 (1980).

[2] S. Hikami, A. I. Larkin, and Y. Nagaoka, Prog. Theor. Phys. 63, 707 (1980).

[3] G. Bergmann, Phys. Rep. 107, 1 (1984).

[4] P. A. Lee, A. D. Stone, and H. Fukuyama, Phys. Rev. B 35, 1039 (1987).

[5] B. L. Altshuler, JETP Lett. 41, 648 (1985).

[6] B. L. Altshuler, and B. Z. Spivak, JETP Lett. 42, 447 (1985).

[7] S. Feng, P. A. Lee, and A. D.Stone, Phys. Rev. Lett. 56, 1960 (1986).

[8] A. D. Stone, Phys. Rev. B 39, 10736 (1989).

[9] A. H. Castro Neto, F. Guinea, N. M. R. Peres, K. S.
Novoselov, and A. K. Geim, Rev. Mod. Phys. 81, 109 (2009).

[10] S. Das Sarma, S. Adam, E. H. Hwang, and E. Rossi, Rev. Mod. Phys. 83, 407 (2010).

[11] H. Suzuura, and T. Ando, Phys. Rev. Lett. 89, 266603 (2002).

[12] D. V. Khveshchenko, Phys. Rev. Lett. 97, 036802 (2006).

[13] P. M. Ostrovsky, I. V. Gornyi, and A. D. Mirlin, Phys. Rev. B 74, 235443 (2006).

[14] E. McCann, K. Kechedzhi, V. I. Fal'ko, H. Suzuura, T. Ando, and B. L. Altshuler, Phys. Rev. Lett. 97, 146805 (2006).

[15] A. F. Morpurgo, and F. Guinea, Phys. Rev. Lett. 97, 196804 (2006).

[16] F. V. Tikhonenko, A. A. Kozikov, A. K. Savchenko, and R. V. Gorbachev, Phys. Rev. Lett. 103, 226801 (2009).

[17] S. V. Morozov, K. S. Novoselov, M. I. Katsnelson, F. Schedin, L. A. Ponomarenko, D. Jiang, and A. K. Geim, Phys. Rev. Lett. 97, 016801 (2006).

[18] K. Kechedzhi, O. Kashuba, and V. I. Fal'ko, Phys. Rev. B 77, 193403 (2008).

[19] M. Y. Kharitonov, and K. B. Efetov, Phys. Rev. B 78, 033404 (2008).

[20] D. W. Horsell, A. K. Savchenko, F. V. Tikhonenko, K. Kechedzhi, I. V. Lerner, and V.I. Fal?ko, Solid State Commun. 149, 1041 (2009).

[21] V. N. Kotov, B. Uchoa, V. M. Pereira, F. Guinea and A. H. Castro-Neto, Rev. Mod. Phys. 84, 1067 (2012).

[22] B. Huard, N. Stander, J. A. Sulpizio, and D. GoldhaberGordon, Phys. Rev. B 78, 121402(R) (2008).

[23] J. H. Scofield, Rev. Sci. Instrum. 58, 985 (1987).

[24] I. Heller, S. Chatoor, J. Männik, M. A. G. Zevenbergen, B. Jeroen, J. B. Oostinga, A. F. Morpurgo, C. Dekker, and S. G. Lemay, Nano Lett. 10, 1563 (2010).

[25] Y. Zhang, E. E. Mendez, and X. Du, ACS Nano 5, 8124 (2011).

[26] G. Xu, C. M. Torres, Jr., Y. Zhang, F. Liu, E. B. Song, M. Wang, Y. Zhou, C. Zeng, and K. L. Wang Nano Lett. 10, 3312 (2010).

[27] N. O. Birge, B. Golding, and W. H. Haemmerle, Phys. Rev. Lett. 62, 195 (1989).

[28] A. Trionfi, S. Lee, and D. Natelson, Phys. Rev. B 70, 041304(R) (2004).

[29] P. Debray, J.-L.Pichard, J. Vicente and P. N. Tung, Phys. Rev. Lett. 63, 2264 (1989).

[30] J. S. Moon, N. O. Birge, and B. Golding, Phys. Rev. B 53, R4193 (1996).

[31] V. Skákalová, A. B. Kaiser, J. S. Yoo, D. Obergfell, and S. Roth, Phys. Rev. B 80, 153404 (2009).

[32] J. Martin, et al. Nature Physics 4, 144 (2008).

[33] V. V. Cheianov and V. I. Fal?ko, Phys. Rev. B 74, 041403(R)(2006).

[34] V. V. Cheianov, V. I. Falko, B. L. Alshuler and I. L. Aleiner, Phys. Rev. Lett. 99, 176801 (2007).

[35] A. Rahman, J. W, Guikema, S. H. Lee and N. Markovic, Phys. Rev. B 87, 081401(R)(2013).

[36] J.-H. Chen, W. G. Cullen, C. Jang, M. S. Fuhrer, and E. D. Williams, Phys. Rev. Lett. 102, 236805 (2009).

[37] Y. Zhang, V. W. Brar, C. Girit, A. Zettl, and M. F. Crommie, Nat. Phys. 5, 722 (2009).

[38] Suyong Jung et al., Nat. Phys. 7, 245 (2011). 